Accepted after revision: January 24, 2011

\title{
Characterization of a Three-Dimensional Mucosal Equivalent: Similarities and Differences with Native Oral Mucosa
}

\author{
Wendy M.W. Tra ${ }^{a}$ Johan W. van Neck ${ }^{a}$ Steven E.R. Hovius ${ }^{a}$ \\ Gerjo J.V.M. van Osch ${ }^{\text {b, c }}$ Soledad Perez-Amodio ${ }^{a}$ \\ Departments of a Plastic and Reconstructive Surgery, ${ }^{b}$ Otorhinolaryngology and ${ }^{\mathrm{c} O r t h o p a e d i c s, ~ E r a s m u s ~ M C, ~}$ \\ Rotterdam, The Netherlands
}

\section{Key Words}

Tissue engineering $\cdot$ Oral mucosa $\cdot$ Keratinocyte $\cdot$

Fibroblast

\begin{abstract}
The aim of this study was to create and characterize a tissueengineered mucosal equivalent (TEM) that closely resembles native mucosa. TEM consists of human primary keratinocytes and fibroblasts isolated from biopsies taken from healthy donors and seeded onto a de-epidermized dermis and cultured for 14 days at the air/liquid interface. The structure of TEM was examined and compared with native nonkeratinizing oral mucosa (NNOM). The various components of the newly formed epidermal layer, basement membrane and underlying connective tissue were analyzed using immunohistochemistry. The mucosal substitute presented in this study showed a mature stratified squamous epithelium that was similar to that of native oral mucosa, as demonstrated by K19, desmoglein-3 and involucrin staining. In addition, the expression of basement membrane components collagen type IV, laminin- 5 and integrin $\alpha 6$ and $\beta 4$ in TEM proved to be consistent with native oral mucosa. The ex-
\end{abstract}

pression of PAS, Ki67, K10 and K13, however, appeared to be different in TEM compared to NNOM. Nevertheless, the similarities with native oral mucosa makes TEM a promising tool for studying the biology of mucosal pathologies such as oral mucositis or fibrosis as well as the development of new therapies.

Copyright $\odot 2011$ S. Karger AG, Basel

\begin{tabular}{ll}
\hline Abbreviations used in this paper \\
\hline BM & basement membrane \\
BSA & bovine serum albumin \\
DED & de-epidermized dermis \\
EDTA & ethylenediaminetetraacetic acid \\
EGF & epidermal growth factor \\
FCS & fetal calf serum \\
KGF & keratinocyte growth factor \\
NNOM & nonkeratinizing native oral mucosa \\
PAS & periodic acid-Schiff \\
PBS & phosphate-buffered saline \\
PI & proliferation index \\
TEM & tissue-engineered mucosal substitutes \\
\hline
\end{tabular}

\section{KARGER}

Fax +41613061234 E-Mail karger@karger.ch www.karger.com (c) 2011 S. Karger AG, Basel

$1422-6405 / 12 / 1953-0185 \$ 38.00 / 0$

Accessible online at:

www.karger.com/cto
Wendy M.W. Tra

Department of Plastic and Reconstructive Surgery, Erasmus MC

Room Ee 15.93, PO Box 2040

NL-3000 CA Rotterdam (The Netherlands)

Tel. +31 10704 3788, E-Mail w.tra@erasmusmc.nl 


\section{Introduction}

Tissue engineering of oral mucosa is a promising technique for studying the biology and pathology of oral mucosa, and can be used as a model to study the dynamics of wound healing [Boyce and Warden, 2002], fibrosis [Wang et al., 2009] and to test cytotoxicity and the working mechanisms of new treatments [Klausner et al., 2007]. Next to these in vitro applications, tissue-engineered mucosal constructs might be used in a clinical setting to provide an alternative source for mucosal grafts for the reconstruction of large oral defects which form a major challenge in reconstructive surgery [Lauer and Schimming, 2001; Izumi et al., 2003].

To serve the above-described purposes, tissue-engineered mucosal substitutes (TEM) should mimic native oral mucosa. Structural features of the tissue such as the presence of a multilayered epidermis, basal layer, basement membrane (BM) and underlying connective tissue as well as the expression pattern of cytokeratins should resemble that of native oral mucosa. Several studies have reported the development of mucosal substitutes, but only few of them included a histological characterization of the construct [Izumi et al., 2004; Rakhorst et al., 2006; Kinikoglu et al., 2009]. Izumi et al. [2004] described the proliferative capacity and keratin 10 (K10)/13 (K13) expression of their mucosal substitutes, and Garzon et al. [2009] reported on the cytokeratin pattern in tissue-engineered periodontal mucosa. Other studies described the pattern of cytokine release of mucosal substitutes [Xu et al., 2009] or the use of a variety of scaffolds to create TEM [Moharamzadeh et al., 2008]. All these studies focussed on some components of the construct, but to our knowledge none of them describe a detailed characterization of the mucosal construct.

The aim of the work described here was to engineer and thoroughly characterize a mucosal substitute that resembles nonkeratinizing native oral mucosa (NNOM). This TEM consists of primary human keratinocytes and fibroblasts isolated from oral mucosa and seeded onto de-epidermized dermis (DED). DED was chosen over collagen-based scaffolds for its resemblance to native connective tissue, easy handling and noncontractile nature. The incorporation of fibroblasts into the scaffold has been proven to be essential for epidermal development [El Ghalbzouri et al., 2002a], as they stimulate keratinocyte proliferation and migration by paracrine mechanisms [El Ghalbzouri et al., 2002b]. The TEM construct was characterized by studying the expression of several components of the BM, the expression pattern of keratins, keratinocyte proliferation and differentiation. As cell-cell adhesion and epidermal attachment to the underlying connective tissue has been shown to be essential for functionality [Green and Jones, 1996], the expression of desmosomes and hemidesmosomes was also evaluated.

\section{Materials and Methods}

Chemicals and Culture Media

Dulbecco's modified Eagle medium $4.5 \mathrm{~g} / 1$ glucose (DMEM), Ham's F12 culture medium, penicillin, streptomycin, gentamicin, amphoceterin B, dispase, collagenase type I and trypsin/ethylenediaminetetraacetic acid (EDTA) were purchased from Invitrogen (Breda, The Netherlands). Fetal calf serum (FCS) was purchased from PAA Laboratories (Cölbe, Germany). Bovine serum albumin, epidermal growth factor (EGF), keratinocyte growth factor (KGF) and other chemicals were purchased from Sigma-Aldrich (Zwijndrecht, The Netherlands). Insulin was purchased from Eli Lilly (Houten, The Netherlands).

\section{Cell Culture}

For this study punch biopsies were taken from healthy individuals, 3 males and 1 female, all Caucasian, aged $62.75 \pm 6.39$ years, upon approval by the Medical Ethics Committee (No. MEC $+2007-282$ ). Half of the biopsies were snap frozen with liquid nitrogen. The remaining biopsies were rinsed thoroughly with phosphate-buffered saline (PBS) and DMEM. The epidermis was separated from the dermis by an overnight incubation in $2.5 \mathrm{mg} /$ $\mathrm{ml}$ dispase solution. After rinsing the epidermal sheet with PBS, keratinocytes were isolated from the epidermis using 0.25\% trypsin-EDTA and the single cell suspension was seeded onto lethally irradiated 3T3 fibroblast feeder layers according to the Rheinwald and Green protocol [Rheinwald and Green, 1975a, b]. Culture medium A consisted of a 3:1 mixture of DMEM and Ham's F12 medium with 5\% FCS, $1 \mu \mathrm{M}$ hydrocortisone, $1 \mu \mathrm{M}$ isoproterenol, 0.1 $\mu \mathrm{M}$ insulin, $100 \mathrm{IU} / \mathrm{ml}$ penicillin, $100 \mu \mathrm{g} / \mathrm{ml}$ streptomycin, and $1 \mathrm{ng} / \mathrm{ml}$ EGF. Fibroblasts were isolated by mincing the dermis followed by incubation in collagenase/dispase $(1.5 \mathrm{mg} / \mathrm{ml} / 2.5 \mathrm{mg} /$ $\mathrm{ml}$, respectively) solution for $2 \mathrm{~h}$ at $37^{\circ} \mathrm{C}$. Next, the collagenase/ dispase solution containing the dermis was filtered twice with a $100-\mu \mathrm{m}$ cell strainer (Sigma-Aldrich), in order to obtain a single cell suspension. The isolated fibroblasts were cultured in fibroblast medium consisting of DMEM supplemented with $10 \%$ FCS, $100 \mathrm{IU} / \mathrm{ml}$ penicillin and $100 \mu \mathrm{g} / \mathrm{ml}$ streptomycin. The cells used in this study were within passage 3-6.

\section{De-Epidermized Dermis}

Human cadaver skin, cryopreserved in $10 \%$ glycerol, and tested negative for cytomegalovirus, human immunodeficiency virus and hepatitis B, was obtained from the European Skin Bank (Beverwijk, The Netherlands). The epidermis was removed from the dermis by gently shaking the skin in PBS supplemented with 200 $\mathrm{IU} / \mathrm{ml}$ penicillin, $200 \mu \mathrm{g} / \mathrm{ml}$ streptomycin and $5 \mu \mathrm{g} / \mathrm{ml}$ amphotericin B. The skin was kept in the PBS solution for 3 weeks and PBS was changed 3 times a week. 
Table 1. Primary antibodies used for immunohistochemistry

\begin{tabular}{lll}
\hline Antibody & Concentration & Source \\
\hline Collagen type IV & $1 / 500$ & Euro-Diagnostica, Nijmegen, The Netherlands \\
Desmoglein-3 & $1 / 500$ & Novus Biologicals, LLC., Littleton, Colo., USA \\
Integrin $\alpha 6$ & $1 / 1,000$ & Novus Biologicals, LLC., Littleton, Colo., USA \\
Integrin $\beta 4$ & $1 / 2,000$ & Novus Biologicals, LLC., Littleton, Colo., USA \\
Involucrin & $1 / 500$ & Novus Biologicals, LLC., Littleton, Colo., USA \\
K10 & $1 / 200$ & Euro-Diagnostica, Nijmegen, The Netherlands \\
K13 & $1 / 200$ & Euro-Diagnostica, Nijmegen, The Netherlands \\
K19 & $1 / 500$ & Novus Biologicals, LLC., Littleton, Colo., USA \\
Ki67 & $1 / 200$ & DAKO, Glostrup, Denmark \\
Laminin-5 & $1 / 1,000$ & Abcam, Cambridge, Mass., USA \\
Vimentin & $1 / 200$ & Euro-Diagnostica, Nijmegen, The Netherlands \\
\hline
\end{tabular}

\section{Tissue-Engineered Mucosa}

TEM was created by spinning $1 \times 10^{5}$ fibroblasts gently into the lamina propria of the DED. For this procedure we used $15-\mathrm{ml}$ tubes filled with $13 \mathrm{ml} \mathrm{1 \%}$ agarose gel (Sigma-Aldrich) on which the DED scaffold is placed, lamina propria side upwards. Next the fibroblasts are placed onto the DED. The tubes containing the DED and fibroblasts are placed in a centrifuge and the fibroblasts are gently spinned into the DED, at $500 \mathrm{rpm}$ for $60 \mathrm{~min}$. Next, the DED containing the fibroblasts was placed onto a stainless steel grid and kept at an air/liquid interface with fibroblast medium for approximately $5 \mathrm{~h}$ to ensure cell attachment to the DED. Finally, the DED was flipped over and the keratinocytes were seeded onto the $\mathrm{BM}$, and kept in a submerged condition for $24 \mathrm{~h}$ with medium A supplemented with $4 \mathrm{ng} / \mathrm{ml}$ KGF instead of EGF. After $24 \mathrm{~h}$, the construct was raised to the air/liquid interface and medium $A$ was replaced by medium B consisting of a 3:1 mixture of DMEM and Ham's F12 supplemented with 1\% FCS, $24 \mu \mathrm{M}$ bovine serum albumin, $1 \mu \mathrm{M}$ hydrocortisone, $1 \mu \mathrm{M}$ isoproterenol, $0.1 \mu \mathrm{M}$ insulin, $10 \mu \mathrm{M} \mathrm{L}$-carnitine, $10 \mathrm{mM}$ L-serine, $1 \mu \mathrm{M} \mathrm{DL}-\alpha$-tocopherolacetate, fatty acid cocktail (15 $\mu \mathrm{M}$ linoleic acid, $7 \mu \mathrm{M}$ arachidonic acid and $25 \mu \mathrm{M}$ palmitic acid), $100 \mathrm{IU} / \mathrm{ml}$ penicillin, $100 \mu \mathrm{g} / \mathrm{ml}$ streptomycin, and $4 \mathrm{ng} / \mathrm{ml} \mathrm{KGF}$. Medium B was removed after $48 \mathrm{~h}$ and replaced by medium $\mathrm{C}$ consisting of medium $\mathrm{B}$ in which FCS was omitted, linoleic acid concentration was increased to $30 \mu \mathrm{M}$ and $50 \mu \mathrm{g} / \mathrm{ml}$ ascorbic acid was added. Medium was changed 3 times a week and TEM construct was harvested after 14 days. In this study, keratinocytes and fibroblasts isolated from biopsies from different donors were never pooled.

\section{Morphology and Immunohistochemistry}

Harvested TEM and NNOM biopsies were snap frozen using liquid nitrogen. Sections $(6 \mu \mathrm{m})$ were cut, and overall morphology was assessed by hematoxylin-eosin and periodic acid-Schiff (PAS) stain. For immunohistochemical analysis of epidermal components K10, K13 and keratin 19 (K19), cryosections were air dried and fixed for $10 \mathrm{~min}$ in acetone. After incubation with the primary antibodies, frozen sections were incubated with Link/ Label complex (BioGenex, San Ramon, Calif., USA) according the manufacturer's instructions. Finally, sections were stained with new fuchsine substrate and hematoxylin. For immunohistochemical analysis of the BM and underlying connective tissue, frozen sections were stained for collagen type IV, laminin, involucrin, vimentin, desmoglein-3, integrin $\alpha 6$ and $\beta 4$ (for antibodies and concentrations used in this study see table 1). After incubation with the primary antibodies, sections were incubated with secondary antibody biotin-labelled goat anti-mouse of goat anti-rabbit (both $1 / 200$, DAKO), followed by incubation with streptavidin-HRP (DAKO) and diaminobenzidine (DAB) substrate. The presence of proliferative cells was assessed using Ki67 antibody, using the protocol as described above. All sections were counterstained with hematoxylin. Controls were included with each staining performed. As a negative control, the primary antibody was replaced by PBS. For the positive control cryosections of normal oral mucosa were used. An isotope control was also included, for which the primary antibody was replaced by mouse anti-human IgG or rabbit anti-human IgG (DAKO), depending on the origin of the primary antibody it replaced. The concentration was similar to that of the primary antibody.

\section{Epidermal Thickness}

Average epidermal thickness was determined by measuring the epidermis at 12 randomly selected regions for both NNOM and TEM, using the NDPI Viewer (Hamamatsu Photonics, Herrsching, Germany). This was repeated for 4 independent experiments, which were done in triplicate. Statistical significance was determined using the Mann-Whitney U test.

\section{Quantification of Cell Proliferation}

To determine the proliferation index (PI), the basal layer of the epithelium was analyzed. Images were taken from 12 randomly chosen microscopic views using $100 \times$ magnification. The PI was established as the ratio of the positive cells to all cells of the basal layer $(\times 100 \%)$, and results were displayed as mean \pm SD. Experiments were done in triplicate. Statistical significance was determined using the Mann-Whitney U test.

\section{Results}

\section{Morphology}

The overall morphology of TEM was assessed by hematoxylin-eosin staining. The newly developed TEM construct had a well-developed, multilayered and stratified squamous epithelium, consisting of a basal, inter- 


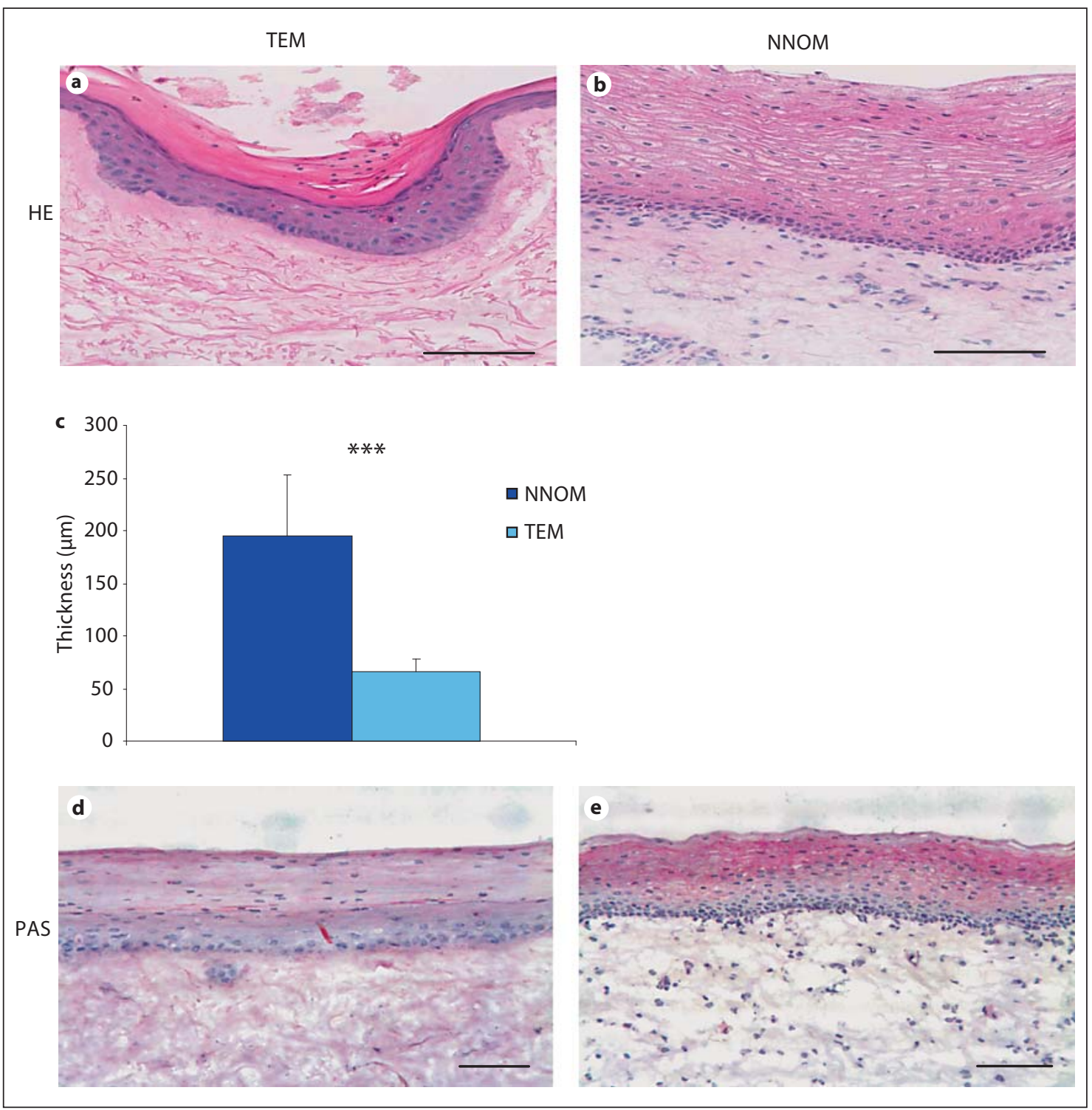

Fig. 1. Hematoxylin-eosin staining of a cross section of TEM (a) and NNOM (b). TEM resembles overall morphology of NNOM, and shows a well-formed, multilayered, parakeratinized epidermis. The epidermis was significantly thinner in TEM (72.6 \pm $26.9 \mu \mathrm{m})$ when compared to NNOM $(194.3 \pm 59.3 \mu \mathrm{m})$. Data are presented as mean $\pm \mathrm{SD}\left({ }^{* * *} \mathrm{p}<0.001\right)(\mathbf{c})$. The keratinocytes in the intermediate layer seemed to have vacuoles. PAS staining showed lower glycogen content in TEM (d) compared with NNOM (e). All pictures are representative for the staining performed. Scale bars $=100 \mu \mathrm{m}$. mediate and superficial layer (fig. 1a) and resembles NNOM (fig. 1b). The epidermis of TEM was parakeratinized, as pyknotic cells were present in the epithelial layer. The basal layer was neatly arranged in two to three layers with cuboidal-shaped cells. The epidermal layer of NNOM has an average thickness of $194.3 \pm 59.3 \mu \mathrm{m}$ and is significantly thicker compared to the epidermis of TEM, which has an average thickness of $72.6 \pm 26.9 \mu \mathrm{m}$ (fig. 1c) $(\mathrm{p}<0.001)$. Vacuoles were observed in the epithelial cells in the intermediate layer of NNOM and TEM. The glycogen content in NNOM was indicated by PAS staining, showing glycogen in the intermediate layer (fig. 1e). PAS staining was also present in the intermediate layer in TEM albeit less intense than in NNOM (fig. 1d). 


\section{Epidermal Differentiation}

Keratin expression in the epidermis was assessed by staining for K10, K13 and K19. TEM showed expression of K10 in the superficial layer only, whereas no expression of K10 was seen in NNOM (fig. 2a, b). K13 stained weakly in the intermediate layer of TEM (fig. 2d), and in both the intermediate and superficial layers of the epithelium of NNOM (fig. 2e). Expression of K19 was observed in the basal layer only of the epithelium of TEM and NNOM (fig. 2g, h). Close observation showed that not every basal cell is positive for K19. Analysis of the basal layer of TEM and NNOM showed that TEM has an average of $74.85 \pm 12.84$ positive cells, which is significantly lower compared to NNOM $(88.03 \pm 6.41 ; \mathrm{p}<0.001)$. Involucrin was observed in all layers except the basal layer in both TEM and NNOM (fig. 2j, k).

\section{Proliferation}

Cells in the basal layer are typically the only cells that proliferate in the epithelium. Proliferation was assessed by Ki67, a protein present in all proliferating cells. In both TEM only (fig. 3a) and NNOM, proliferating cells were observed in the basal layer only (fig. 3b). The PI was significantly lower in TEM $(36.6 \pm 11.1)$ compared to $\operatorname{NNOM}(55.8 \pm 8.3)$ (fig. $3 \mathrm{~d})(\mathrm{p}<0.001)$

\section{Connective Tissue}

Fibroblasts are the predominant cell type in the dermal layer. The presence and distribution of fibroblasts were assessed by vimentin staining. In TEM, fibroblasts were found in the lamina propria, where they were mainly located on the bottom surface of the lamina propria (fig. 3e). In NNOM, the fibroblasts were found distributed homogeneously in the connective tissue (fig. 3f).

\section{Basement Membrane}

The presence of BM components collagen type IV and laminin-5 were first determined in the DED scaffold. Collagen type IV is a component of basal lamina and laminin-5 is a component of the anchoring filaments. Both components were expressed in the scaffold along the whole dermal-epidermal junction before seeding of fibroblasts and keratinocytes (fig. 4a-e). The expression of collagen type IV and laminin-5 was restricted to the $\mathrm{BM}$. The addition of fibroblasts and keratinocytes did not alter the expression of collagen type IV (fig. $4 \mathrm{~b}$ ) or laminin-5 (fig. 4f) in TEM. Collagen type IV expression was higher in TEM (fig. 4b) than in NNOM (fig. 4c). Laminin-5 was more abundantly expressed in NNOM (fig. 4g) than in TEM (fig. 4f).

Tissue-Engineered Mucosa

\section{Dermal-Epidermal Junction}

Desmosomes and hemidesmosomes are responsible for cell-cell attachment and the attachment of the epithelial layer to the underlying connective tissue. Cell-cell attachment by desmosomes was assessed by desmoglein-3, a glycoprotein component of desmosomes. Cells in the basal and intermediate layer of TEM and NNOM were equally positive for desmoglein-3 (fig. $5 \mathrm{a}, \mathrm{b}$ ). Before the seeding of fibroblasts and keratinocytes, the hemidesmosomal subunits integrin $\alpha 6$ and $\beta 4$ were not present in the DED scaffold (fig. $5 \mathrm{~d}$, e). Integrin $\alpha 6$ was expressed along the whole dermal-epidermal junction, predominantly in the BM, in both TEM (fig. 5f) and NNOM (fig. 5e). Integrin $\beta 4$ was also present along the whole dermal-epidermal junction, predominantly in the BM of both TEM and NNOM, although the intensity of integrin $\beta 4$ was lower in TEM compared to NNOM. In all sections used, the newly formed epithelium was attached to the underlying connective tissue and detachment was never seen.

\section{Discussion}

The aim of this study was to create a mucosal construct that resembles native oral mucosa. Therefore, we engineered TEM by seeding oral keratinocytes and fibroblasts onto DED. Next, TEM and NNOM were compared on epidermal structure, BM and underlying connective tissue organization. Here we show an oral mucosal construct composed of a multilayered epithelium with many morphological and immunological characteristics observed in NNOM. No morphological differences were observed between the TEM constructs from different donors. K19, Ki67, involucrin, integrin $\alpha 6$ and $\beta 4$, vimentin, collagen type IV and laminin-5 were present in our TEM construct (table 2). Overall morphology of TEM was observed to be similar to NNOM, although the epidermal layer of TEM was found to be significantly thinner compared to NNOM. Despite the epidermis being thinner in TEM, essential components such as a BM, a proliferating basal layer and a differentiated epidermis were present in the epidermal layer and resembled NNOM. The presence of involucrin, a marker for terminal differentiation [Alaminos et al., 2007], demonstrated that a mature epithelium was formed in TEM. The DED scaffold used in this study was chosen for its easy handling, noncontractile nature and firmness. The scaffold has to be able to withstand mechanical stress, as the oral mucosa is continuously exposed to some degree of mechanical stress [Pres-

Cells Tissues Organs 2012;195:185-196 


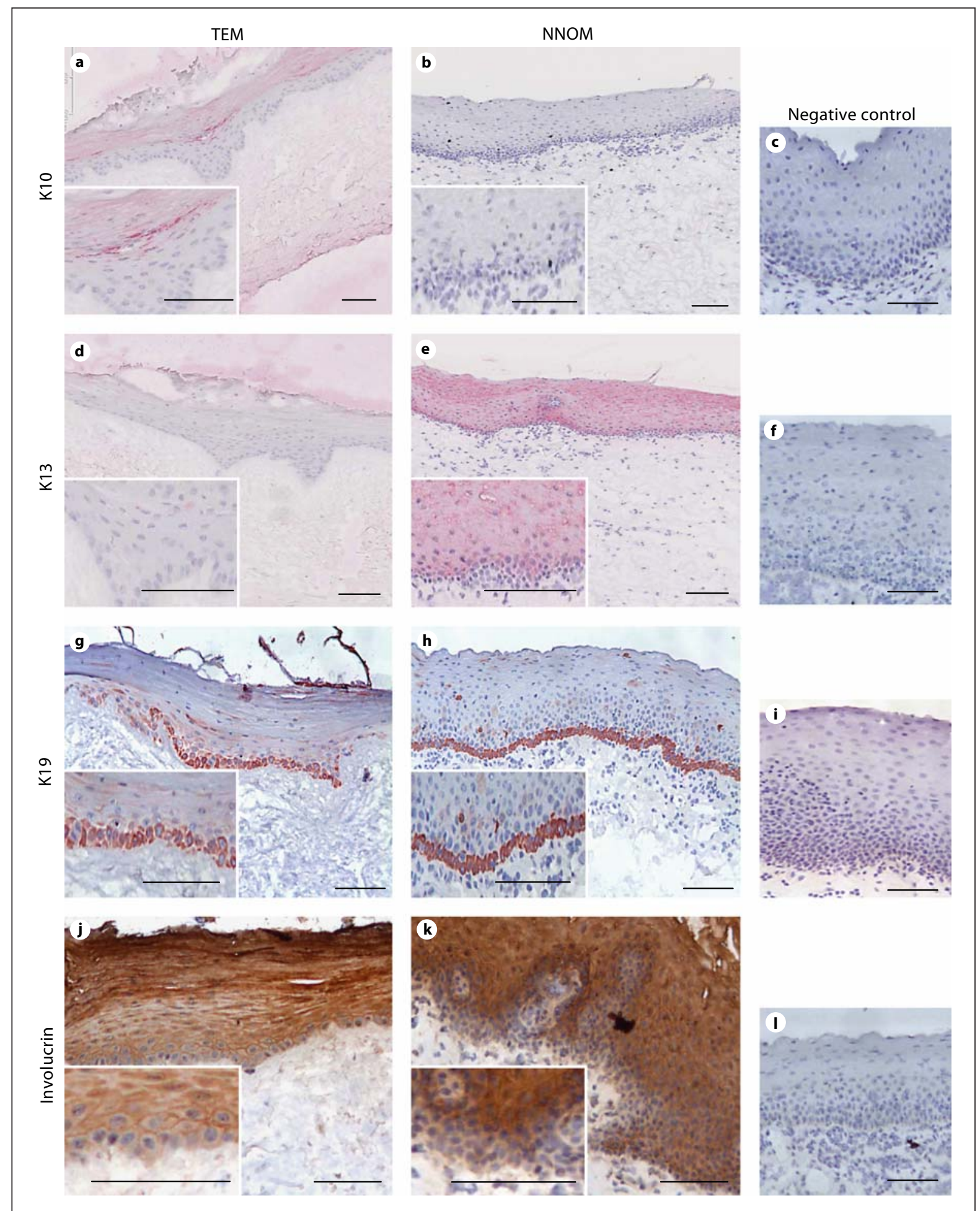

Fig. 2. Keratin expression was assessed in TEM and NNOM. K10, present in keratinized mucosa, was present in the superficial layer of the epidermis of TEM (a) but was not observed in NNOM (b). $\mathrm{K} 13$, present in nonkeratinizing epithelium, was weakly expressed in TEM (d) but was strongly expressed in $\operatorname{NNOM}(\mathbf{e})$. K19, present in the basal layer of nonkeratinizing epithelium of the oral cavity, was observed in the basal layer of TEM (g) and NNOM (h). The expression pattern of involucrin in TEM (j) proved to be similar to $\operatorname{NNOM}(\mathbf{k})$. c, f, i, I Negative controls of all stainings are included. All pictures are representative for the stainings. Scale bars $=100$ $\mu \mathrm{m}$. 


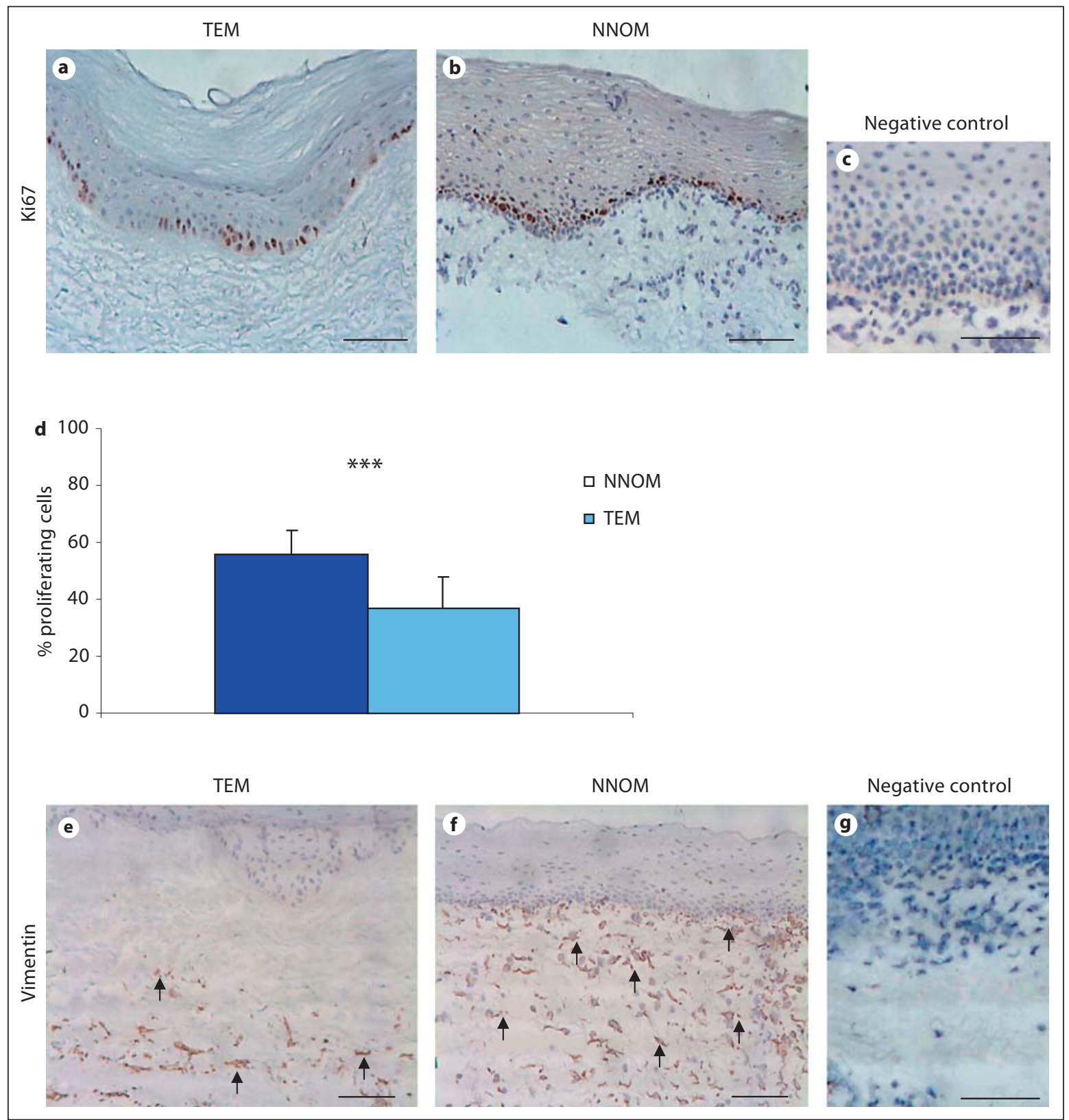

Fig. 3. Cell proliferation in TEM (a) was compared to NNOM (b). Proliferating cells were only observed in the basal layer of both NNOM and TEM. The number of proliferating cells in TEM (36.6 $\pm 11.1)$ was significantly lower compared to NNOM (558 \pm 8.3$)$. Data are presented as mean \pm SD $\left({ }^{* *} \mathrm{p}<0.001\right)(\mathbf{d})$. Fibroblast distribution was assessed by vimentin staining. In TEM, fibro-

land and Jurevic, 2002]. We also found the presence of $\mathrm{BM}$ components an advantage as the BM connects the epithelium to the underlying connective tissue. The seeded cells do not have to deposit these BM components themselves before developing the new epithelial layer. blasts (indicated by arrows) were seen only on the lower surface of the lamina propria (e), whereas in NNOM, fibroblasts were seen throughout the connective tissue (f). Negative controls of both stainings are included $(\mathbf{c}, \mathbf{g})$. All pictures shown are representative for the stainings. Scale bars $=100 \mu \mathrm{m}$.

The oral epithelium generally has a high number of proliferating cells. In buccal mucosa the turnover rate is approximately 2-3 times that of the epidermis of the skin [Winning and Townsend, 2000], and a PI of 58.8 has been reported in native buccal mucosa [van Oijen et al., 1998]. 


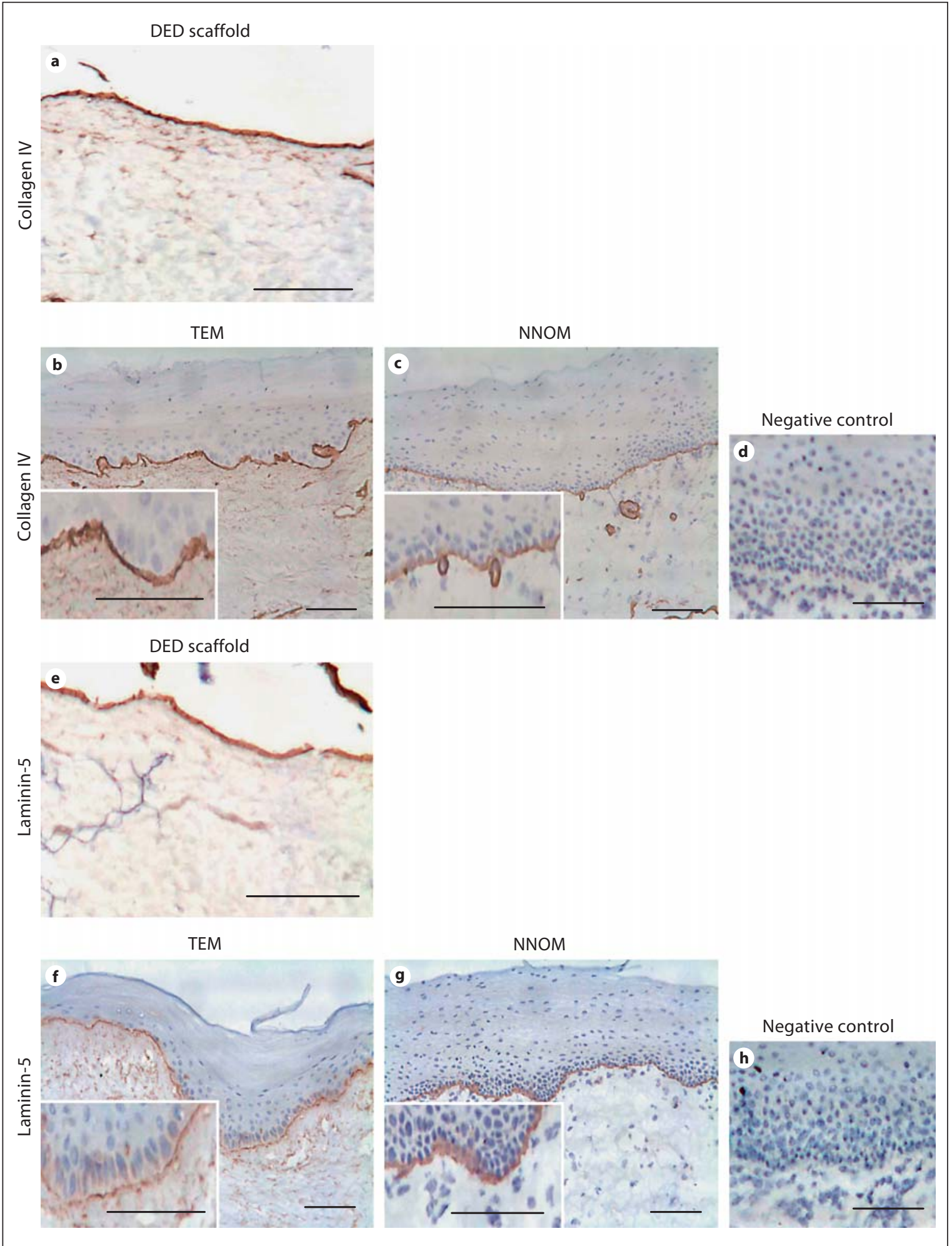

Fig. 4. BM components collagen type IV (a) and laminin-5 (e) were present in the DED scaffold before seeding of keratinocytes and fibroblasts. Collagen type IV and laminin-5 were equally expressed in TEM (b, f) and NNOM $(\mathbf{c}, \mathbf{g})$. Negative controls are included $(\mathbf{d}, \mathbf{h})$. All pictures shown are representative for the stainings. Scale bars = $100 \mu \mathrm{m}$. 

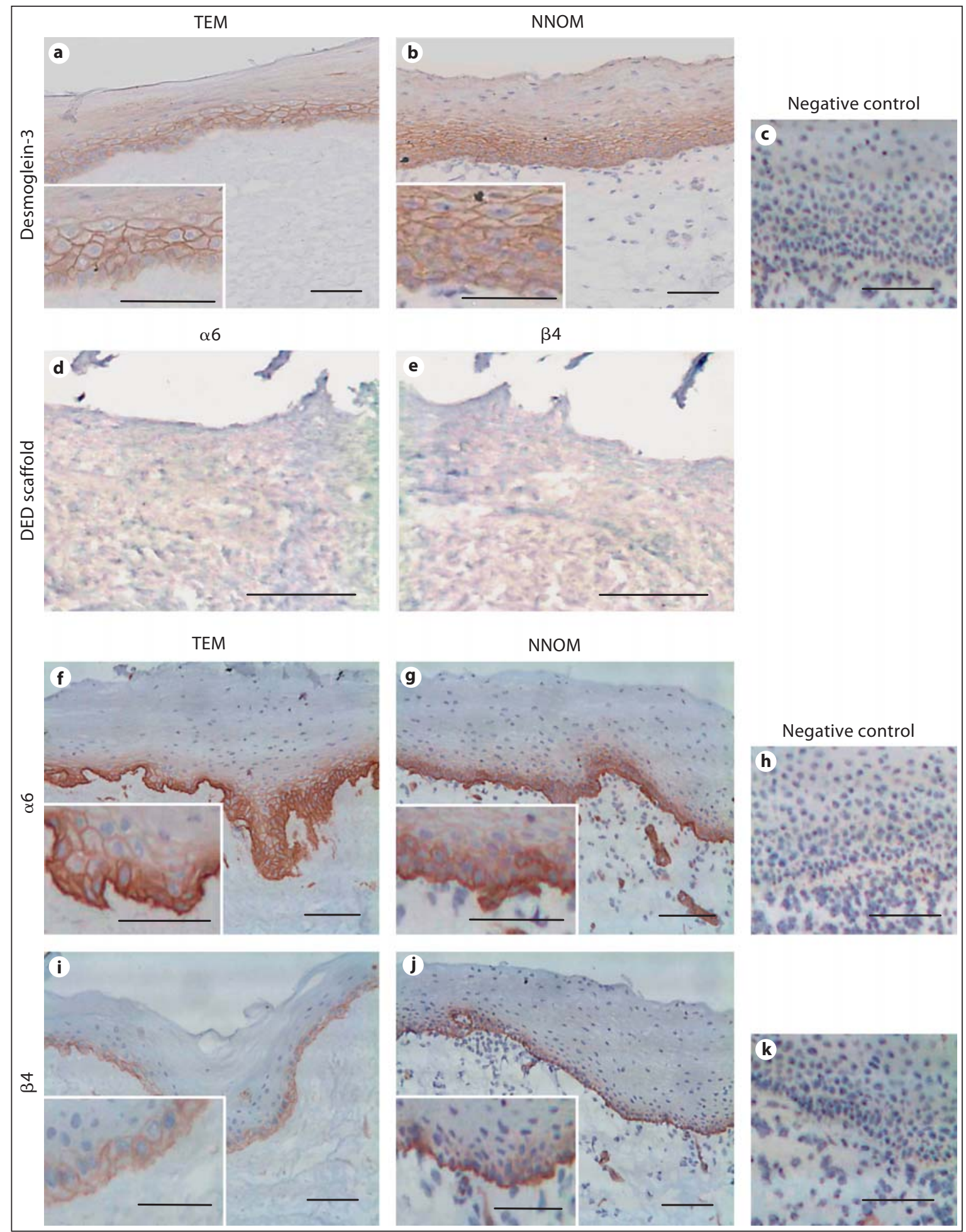

Fig. 5. Cell-cell adhesion was assessed by desmoglein-3, a glycoprotein of desmosomes. In both TEM (a) and NNOM (b) desmoglein-3 was observed in the basal layers of the epidermis. Hemi-desmosomal subunits $\alpha 6$ and $\beta 4$ were not expressed in the DED scaffold (d, e). A similar expression pattern for integrin $\alpha 6$ was observed in TEM (f) and NNOM (g). Integrin $\beta 4$ expression was seen in the basal layer of TEM (i) and NNOM (j), although the intensity in TEM was lower than in NNOM. c, h, k Negative controls are included. All pictures shown are representative for the stainings. Scale bars $=100 \mu \mathrm{m}$. 
Table 2. Summary of epidermal characteristics of TEM and NNOM

\begin{tabular}{lll}
\hline & \multicolumn{2}{l}{ Epidermis } \\
\cline { 2 - 3 } & TEM & NNOM \\
\hline Tissue architecture & $\mathrm{BL}, \mathrm{IL}, \mathrm{SL}$ & $\mathrm{BL}, \mathrm{IL}, \mathrm{SL}$ \\
Number of living cell layers & $5-10$ & $15-20$ \\
K10 & $\mathrm{SL}$ & $\mathrm{absent}$ \\
K13 & $\mathrm{SL}$ & $\mathrm{IL}, \mathrm{SL}$ \\
K19 & $\mathrm{BL}$ & $\mathrm{BL}$ \\
Ki67 & $\mathrm{BL},++$ & $\mathrm{BL},++++$ \\
Desmoglein-3 & $\mathrm{BL}, \mathrm{IL}$ & $\mathrm{BL}, \mathrm{IL}$ \\
Integrin $\alpha 6$ & $\mathrm{BL}$ & $\mathrm{BL}$ \\
Integrin $\beta 4$ & $\mathrm{BL}$ & $\mathrm{BL}$ \\
Collagen type IV & $\mathrm{BL}$ & $\mathrm{BL}$ \\
Laminin-5 & $\mathrm{BL}$ & $\mathrm{BL}$ \\
Involucrin & $\mathrm{BL}, \mathrm{IL}, \mathrm{SL}$ & $\mathrm{BL}, \mathrm{IL}, \mathrm{SL}$ \\
\hline
\end{tabular}

$\mathrm{BL}=$ Basal layer $; \mathrm{IL}=$ intermediate layer $\mathrm{SL}=$ superficial layer.

After 3 weeks of culture, our TEM model showed proliferating cells in the basal layer of the construct, indicating a self-renewal capacity of TEM. The PI of TEM was found to be significantly lower than that of NNOM. The lower PI of TEM was in accordance with the lower staining intensity of PAS indicating less glycogen storage in TEM than in NNOM. Many differences have been reported about the number of proliferating cells in mucosal equivalents. Some studies report hyperproliferation [Chung et al., 1997; Izumi et al., 2004], slightly reduced proliferation [Kinikoglu et al., 2009] or strongly reduced to no proliferation [Pena et al., 2010]. It has been shown that the number of keratinocytes expressing Ki67 progressively decreases over time, showing maximum proliferative capacity in the first week of culture which decreases to a few cells at the end of the third week of culture [Tomakidi et al., 1998; Kinikoglu et al., 2009]; the latter is in accordance with our observations. The discrepancies between the studies might be a result of the differences in scaffold, which varies from DED to fibrin, and the composition of the culture media used to construct the mucosal substitutes, which are very variable between different studies.

Keratins are the predominant cytoskeletal proteins in epithelia, and depending on the stage of differentiation and cell type different cytokeratins are expressed [Presland and Jurevic, 2002]. The differentiation pattern of our TEM construct was assessed by K10, K13 and K19. K19 was observed in the majority of the cells of the basal layer of the epithelium of TEM, indicating a nonkeratinizing nature of the epithelium of our construct. K13, a keratin present in the intermediate and superficial layer of the nonkeratinizing epithelium, was weakly expressed in TEM. Interestingly, K10, an early differentiation marker of keratinizing epithelium, was found to be expressed in the superficial layer of the epidermis in TEM, which is unusual as K10 is normally expressed in the spinous layer of keratinizing epithelia. We hypothesize that this K10 expression in the superficial layer of TEM is the result of the fact that we handle TEM gently and apply minimal mechanical trauma, meaning that this layer was never rubbed off, as would happen in native oral epithelium.

Previous studies have shown that connective tissues are responsible for the final pattern of oral epithelium differentiation [Karring et al., 1975; Mackenzie and Hill, 1984; Chung et al., 1997]. The DED scaffold used to create the TEM construct originates from donor skin, which might explain the keratin expression pattern in TEM. The use of fibroblasts taken from buccal mucosa incorporated in TEM did not lead to direct expression of K13. This suggests that the connective tissue has a higher influence on the differentiation pattern of the epidermis than the incorporated fibroblasts or the seeded keratinocytes. This finding is in line with a study by Rakhorst et al. [2006] which showed that incorporation of fibroblasts in TEM did not affect the differentiation pattern of the keratinocytes. In addition, other studies suggested that intrinsic and extrinsic factors influence the differentiation process of the epithelium and that the expression of $\mathrm{K} 10$ and K13 might be determined by the connective tissue, whereas K19 expression might be determined by the epithelium [Kautsky et al., 1995]. Another explanation for the differences in keratin expression could be the location of the fibroblasts; keratin expression may vary with the distribution of fibroblasts which are, in the TEM model, not present close to the epithelium.

In this study, the presence and distribution of fibroblasts in TEM and NNOM was assessed by vimentin staining. In TEM, fibroblasts were found to be on the lower half of the connective layer, whereas in NNOM they are dispersed homogenously in the connective layer. Several studies have shown the necessity of incorporating fibroblasts into the scaffold for epidermal development, as they stimulate keratinocyte proliferation and migration [El Ghalbzouri et al., 2002a; Rakhorst et al., 2006]. In addition, the presence of fibroblasts is known to lead to a well-developed epidermal layer as these cells secrete growth factors, and the paracrine cross talk between keratinocytes and fibroblasts is essential for the formation of a new epidermis [El Ghalbzouri and Ponec, 2004]. Even 
though the fibroblasts were not homogenously distributed in TEM, the construct displays a well-developed, multilayered epidermis.

Cell-cell adhesion and attachment of the epidermal layer to the connective tissue are essential for correct functionality of NNOM and therefore also for mucosal substitutes [Green and Jones, 1996; Presland and Jurevic, 2002]. Desmosomes are complexes that link the keratinocytes to each other. An important component of desmosomes is the protein desmoglein-3. Immunohistochemistry proved that desmoglein-3 was present in TEM and a similar expression pattern was found in NNOM. This suggests that the cells were actively synthesizing components to ultimately form desmosomes for cell-cell adhesion to maintain structural stability and functionality. Hemidesmosomes are responsible for the adhesion of the epithelial layer to the BM of the connective layer. Integrin $\alpha 6$ and $\beta 4$, subunits of hemidesmosomes, were found to be similarly expressed in NNOM and TEM constructs. This corresponding expression of these integrins and desmosomal protein desmoglein-3 implies that TEM constructs form a number of proteins necessary for the formation of desmosomes and hemidesmosomes in order to form a tight barrier which mimics native oral mucosa and might also mimic its functionality. However, this specific aspect remains to be proven.
The applications for TEM range from transplantation into the oral cavity to studying oral mucositis or fibrosis. These applications require further investigation. Before TEM can be used for transplantation, we have to test it in vivo to determine the quality of TEM posttransplantation by, for example, assessing viability and angiogenesis. To study oral mucositis the TEM construct has to be extended as no immune cells are present in the current model. An addition of immune cells such as dendritic cells or Langerhans cells would be required [Bechetoille et al., 2007], or TEM could be treated with proinflammatory cytokines modifying TEM to create an equivalent mimicking oral mucositis [Tjabringa et al., 2008].

In summary, the TEM construct developed in this study resembles NNOM. TEM is an oral mucosal construct composed of a multilayered epithelium with many morphological and immunological characteristics observed in NNOM. Based on the results of this study, we are convinced that TEM holds promise for future research, for example, the early mechanisms leading to oral mucositis and fibrosis.

\section{Acknowledgement}

This study was supported by a grant from Nuts-OHRA Foundation (No. 0802-105).

\section{References}

Alaminos, M., I. Garzon, M.C. Sanchez-Quevedo, G. Moreu, M. Gonzalez-Andrades, A. Fernandez-Montoya, A. Campos (2007) Time-course study of histological and genetic patterns of differentiation in human engineered oral mucosa. J Tissue Eng Regen Med 1: 350-359.

Bechetoille, N., C. Dezutter-Dambuyant, O. Damour, V. Andre, I. Orly, E. Perrier (2007) Effects of solar ultraviolet radiation on engineered human skin equivalent containing both Langerhans cells and dermal dendritic cells. Tissue Eng 13: 2667-2679.

Boyce, S.T., G.D. Warden (2002) Principles and practices for treatment of cutaneous wounds with cultured skin substitutes. Am J Surg 183: 445-456.

Chung, J.H., K.H. Cho, D.Y. Lee, O.S. Kwon, M.W. Sung, K.H. Kim, H.C. Eun (1997) Human oral buccal mucosa reconstructed on dermal substrates: a model for oral epithelial differentiation. Arch Dermatol Res 289: 677-685.

-El Ghalbzouri, A., S. Gibbs, E. Lamme, C.A. Van Blitterswijk, M. Ponec (2002a) Effect of fibroblasts on epidermal regeneration. $\mathrm{Br} \mathrm{J}$ Dermatol 147: 230-243.
El Ghalbzouri, A., E. Lamme, M. Ponec (2002b) Crucial role of fibroblasts in regulating epidermal morphogenesis. Cell Tissue Res 310: 189-199.

El Ghalbzouri, A., M. Ponec (2004) Diffusible factors released by fibroblasts support epidermal morphogenesis and deposition of basement membrane components. Wound Repair Regen 12: 359-367.

Garzon, I., M.C. Sanchez-Quevedo, G. Moreu, M. Gonzalez-Jaranay, M. Gonzalez-Andrades, A. Montalvo, A. Campos, M. Alaminos (2009) In vitro and in vivo cytokeratin patterns of expression in bioengineered human periodontal mucosa. J Periodontal Res 44: 588-597.

Green, K.J., J.C. Jones (1996) Desmosomes and hemidesmosomes: structure and function of molecular components. FASEB J 10: 871-881.

Izumi, K., S.E. Feinberg, A. Iida, M. Yoshizawa (2003) Intraoral grafting of an ex vivo produced oral mucosa equivalent: a preliminary report. Int J Oral Maxillofac Surg 32: 188197.
Izumi, K., J. Song, S.E. Feinberg (2004) Development of a tissue-engineered human oral mucosa: from the bench to the bed side. Cells Tissues Organs 176: 134-152.

Karring, T., N.P. Lang, H. Loe (1975) The role of gingival connective tissue in determining epithelial differentiation. J Periodontal Res 10: 1-11.

Kautsky, M.B., P. Fleckman, B.A. Dale (1995) Retinoic acid regulates oral epithelial differentiation by two mechanisms. J Invest Dermatol 104: 224-230.

Kinikoglu, B., C. Auxenfans, P. Pierrillas, V. Justin, P. Breton, C. Burillon, V. Hasirci, O. Damour (2009) Reconstruction of a fullthickness collagen-based human oral mucosal equivalent. Biomaterials 30: 6418-6425.

Klausner, M., S. Ayehunie, B.A. Breyfogle, P.W. Wertz, L. Bacca, J. Kubilus (2007) Organotypic human oral tissue models for toxicological studies. Toxicol In Vitro 21: 938-949.

Lauer, G., R. Schimming (2001) Tissue-engineered mucosa graft for reconstruction of the intraoral lining after freeing of the tongue: a clinical and immunohistologic study. J Oral Maxillofac Surg 59: 169-175, discussion 175-167. 
Mackenzie, I.C., M.W. Hill (1984) Connective tissue influences on patterns of epithelial architecture and keratinization in skin and oral mucosa of the adult mouse. Cell Tissue Res 235: 551-559.

-Moharamzadeh, K., I.M. Brook, R. Van Noort, A.M. Scutt, K.G. Smith, M.H. Thornhill (2008) Development, optimization and characterization of a full-thickness tissue engineered human oral mucosal model for biological assessment of dental biomaterials. J Mater Sci Mater Med 19: 1793-1801.

Pena, I., L.M. Junquera, A. Meana, E. Garcia, V. Garcia, J.C. De Vicente (2010) In vitro engineering of complete autologous oral mucosa equivalents: characterization of a novel scaffold. J Periodontal Res 45: 375-380.

Presland, R.B., R.J. Jurevic (2002) Making sense of the epithelial barrier: what molecular biology and genetics tell us about the functions of oral mucosal and epidermal tissues. J Dent Educ 66: 564-574.
Rakhorst, H.A., S.J. Posthumus-Van Sluijs, W.M. Tra, J.W. Van Neck, G.J. Van Osch, S.E. Hovius, A. El Ghalbzouri, S.O. Hofer (2006) Fibroblasts accelerate culturing of mucosal substitutes. Tissue Eng 12: 2321-2331.

Rheinwald, J.G., H. Green (1975a) Formation of a keratinizing epithelium in culture by a cloned cell line derived from a teratoma. Cell 6: 317-330.

Rheinwald, J.G., H. Green (1975b) Serial cultivation of strains of human epidermal keratinocytes: the formation of keratinizing colonies from single cells. Cell 6: 331-343.

Tjabringa, G., M. Bergers, D. van Rens, R. de Boer, E. Lamme, J. Schalkwijk (2008) Development and validation of human psoriatic skin equivalents. Am J Pathol 173: 815-823.

Tomakidi, P., D. Breitkreutz, N.E. Fusenig, J. Zoller, A. Kohl, G. Komposch (1998) Establishment of oral mucosa phenotype in vitro in correlation to epithelial anchorage. Cell Tissue Res 292: 355-366. van Oijen, M.G., M.M. Gilsing, G. Rijksen, G.J. Hordijk, P.J. Slootweg (1998) Increased number of proliferating cells in oral epithelium from smokers and ex-smokers. Oral Oncol 34: 297-303.

Wang, X., Y. Liu, Z. Deng, R. Dong, S. Hu, Y. Li, Y. Jin (2009) Inhibition of dermal fibrosis in self-assembled skin equivalents by undifferentiated keratinocytes. J Dermatol Sci 53: 103-111.

Winning, T.A., G.C. Townsend (2000) Oral mucosal embryology and histology. Clin Dermatol 18: 499-511.

Xu, Q., K. Izumi, T. Tobita, Y. Nakanishi, S.E. Feinberg (2009) Constitutive release of cy tokines by human oral keratinocytes in an organotypic culture. J Oral Maxillofac Surg 67: 1256-1264. 